\title{
FIXED POINT THEOREMS FOR LIPSCHITZIAN PSEUDO-CONTRACTIVE MAPPINGS
}

\author{
JUAN A. GATICA ${ }^{1}$ AND W. A. KIRK ${ }^{2}$
}

\begin{abstract}
Let $X$ be a Banach space and $D \subset X$. A mapping $U: D \rightarrow X$ is said to be pseudo-contractive if, for all $u, v \in D$ and all $r>0,\|u-v\| \leqq\|(1+r)(u-v)-r(U(u)-U(v))\|$. A recent fixed point theorem of W. V. Petryshyn is used to prove: If $G$ is an open bounded subset of $X$ with $0 \in G$ and $U: \bar{G} \rightarrow X$ is a lipschitzian pseudo-contractive mapping satisfying (i) $U(x) \neq \lambda x$ for $x \in \partial G$, $\hat{\imath}>1$, and (ii) $(I-U)(\bar{G})$ is closed, then $U$ has a fixed point in $\bar{G}$. This result yields fixed point theorems for pseudo-contractive mappings in uniformly convex spaces and for "strongly" pseudocontractive mappings in reflexive spaces.
\end{abstract}

Let $X$ be a Banach space and $D \subset X$. A mapping $U: D \rightarrow X$ is said to be pseudo-contractive if, for all $u, v \in D$ and all $r>0$,

$$
\|u-v\| \leqq\|(1+r)(u-v)-r(U(u)-U(v))\| .
$$

A characterization of $F$. Browder given in [1] establishes the importance of this class of mappings; he observes that a necessary and sufficient condition that $U: D \rightarrow X$ be pseudo-contractive is that $I-U$ be accretive.

In this paper we prove a fixed point theorem for such mappings by imposing the Leray-Schauder condition ((i) below) used by Browder [2] in his study of semicontractive mappings. Our results illustrate the firm connection which exists between the lipschitzian pseudo-contractive mappings and the class of nonexpansive mappings. This connection was observed by Kirk in [3], and Theorem 2 below represents a considerable generalization of Theorem 1 of [3] in a slightly more restricted setting.

Throughout the paper we use $\partial A$ to denote the boundary of a set $A \subset X$.

Presented to the Society, March 26, 1971; received by the editors March 18, 1971 and, in revised form, March 13, 1972.

AMS 1969 subject classifications. Primary 4785.

Key words and phrases. Fixed point theorem, pseudo-contractive mapping, nonexpansive mapping, accretive mapping.

${ }^{1}$ Research of the first author was partially supported by the Universidad de Concepción, Concepción, Chile.

${ }^{2}$ Research of the second author was supported by National Science Foundation Grant GP-18054.

(c) American Mathematical Society 1972 
THEOREM 1. Let $X$ be a Banach space, $G$ an open bounded subset of $X$ with $0 \in G$, and let $U: \bar{G} \rightarrow X$ be a lipschitzian pseudo-contractive mapping satisfying:

(i) $U(x) \neq \lambda x$ if $x \in \partial G$ and $\lambda>1$,

(ii) $(I-U)(\bar{G})$ is closed.

Then $U$ has a fixed point in $\bar{G}$.

THEOREM 2. Let $X$ be a uniformly convex Banach space whose conjugate space $X^{*}$ is also uniformly convex, let $G$ be a bounded open convex subset of $X$ with $0 \in G$, and let $U: X \rightarrow X$ be a lipschitzian pseudo-contractive mapping satisfying $U(x) \neq \lambda x$ if $x \in \partial G$ and $\lambda>1$. Then $U$ has a fixed point in $\bar{G}$.

Under the assumptions of Theorem $2, I-U$ is a continuous accretive mapping defined on all of $X$, so by Theorem 4 of Browder [2], $I-U$ is demiclosed. Since $\bar{G}$ is weakly closed, $(I-U)(\bar{G})$ is closed and Theorem 2 follows from Theorem 1 .

Our proof of Theorem 1 makes use of a recent result of W. V. Petryshyn [6, Theorem 7]. With $G$ as in Theorem 1, he has shown that if $T: \bar{G} \rightarrow X$ is a 1-set-contraction (defined in the remark below) satisfying (i) and (ii), then $T$ has a fixed point in $\bar{G}$. Nonexpansive mappings, or more generally the semicontractive mappings of Browder [2], provide important examples of 1-set-contractions. We will apply Petryshyn's result below in cases where either $T$ is a contraction mapping, or $T$ is nonexpansive. Its full generality shows that Theorem 1 holds for the lipschitzian " 1 -set pseudocontractive mappings" as indicated in our final remark.

ProOF OF THEOREM 1. Let $0<r<1$ be chosen so that $r U$ is a contraction mapping. Define mappings $S, T$ of $\bar{G}$ into $X$ by $S=(1-r) I$, $T=I-r U$. Then $T$ is one-to-one, $T(G)$ is open, $\partial T(G)=T(\partial G)$, and thus $T(\bar{G})=\operatorname{cl}(T(G))$. Since $r U$ satisfies (i) and (ii) on $\bar{G}$, by Petryshyn's Theorem there exists $x \in \bar{G}$ such that $x=r U(x)$. Hence $x \in G$ (because $U$ satisfies (i)) and so $0=T(x) \in T(G)$ yielding $0 \in$ int $B$ where $B=\operatorname{cl}(T(G)$ ).

Since $U$ is pseudo-contractive, for each $x, y \in G$,

$$
\begin{aligned}
\|x-y\| & \leqq\|(1+r)(x-y)-r(U(x)-U(y))\| \\
& \leqq\|(x-r U(x))-(y-r U(y))\|+r\|x-y\| ;
\end{aligned}
$$

thus $(1-r)\|x-y\| \leqq\|T(x)-T(y)\|$ which yields

$$
\|S(x)-S(y)\| \leqq\|T(x)-T(y)\|, \quad x, y \in G .
$$

Now define $H: B \rightarrow X$ by $H(z)=S T^{-1}(z)$. Then if $z_{1}, z_{2} \in B$,

$$
\begin{aligned}
\left\|H\left(z_{1}\right)-H\left(z_{2}\right)\right\| & =\left\|S T^{-1}\left(z_{1}\right)-S T^{-1}\left(z_{2}\right)\right\| \\
& \leqq\left\|T T^{-1}\left(z_{1}\right)-T T^{-1}\left(z_{2}\right)\right\|=\left\|z_{1}-z_{2}\right\|,
\end{aligned}
$$

so $H$ is nonexpansive on $B$. 
To see that $(I-H)(B)$ is closed, suppose $z_{n}-H\left(z_{n}\right) \rightarrow y, z_{n} \in B$. Then $z_{n}-(1-r) T^{-1}\left(z_{n}\right) \rightarrow y$ yielding

$$
z_{n} /(1-r)-T^{-1}\left(z_{n}\right) \rightarrow y /(1-r) .
$$

Let $z=y /(1-r)$ and let $x_{n}=T^{-1}\left(z_{n}\right)$. Then

$$
\begin{aligned}
r\left[x_{n}-U\left(x_{n}\right)\right] /(1-r) & =\left[x_{n}-r U\left(x_{n}\right)\right] /(1-r)-x_{n} \\
& =T\left(x_{n}\right) /(1-r)-x_{n} \rightarrow z,
\end{aligned}
$$

and thus $x_{n}-U\left(x_{n}\right) \rightarrow(1-r) z / r$. Since $(I-U)(\bar{G})$ is closed, there exists $x \in \bar{G}$ such that $x-U(x)=(1-r) z / r$. Then

$$
(1-r) z=r(x-U(x))=x-r U(x)-(1-r) x=T(x)-(1-r) x,
$$

yielding $T(x) /(1-r)-x=z$. Letting $w=T(x)$ we have $w /(1-r)-T^{-1}(w)=z$, so $w-(1-r) T^{-1}(w)=(1-r) z=y$. Hence $w-H(w)=y$ and we conclude $(I-H)(B)$ is closed.

Now we show that $H$ satisfies (i) on $B$. Let $x \in \partial B$ and suppose $H(x)=\lambda x$ for some $\lambda>1$. Then $T^{-1}(x)=\lambda x /(1-r)$, and since $T(\partial G)=\partial T(G)$, we conclude $\lambda x /(1-r) \in \partial G$. Thus we have $x=T(\lambda x /(1-r))$, so

$$
x=\lambda x /(1-r)-r U(\lambda x /(1-r)),
$$

which implies $U(\lambda x /(1-r))=(\lambda+r-1) x / r(1-r)$.

Let $\bar{x}=\lambda x /(1-r)$. Then $\bar{x} \in \partial G$ and $U(\bar{x})=\mu \bar{x}$ where $\mu=(\lambda+r-1) / \lambda r$. But $\mu-1=(\lambda-1)(1-r) / \lambda r>0$, and this contradicts our hypothesis (i) for $U$ on $\partial G$.

Therefore, we conclude that $H$ satisfies all the hypotheses for Petryshyn's theorem on $B$, so there exists $y \in B$ such that $H(y)=y$. Letting $x=$ $T^{-1}(y), S(x)=S T^{-1}(y)=H(y)=y=T(x)$; thus $(1-r) x=x-r U(x)$ and we have $U(x)=x$ completing the proof.

We say that a mapping $U: X \rightarrow X$ is strongly pseudo-contractive relative to $D \subset X$ if for each $x \in X$ and $r>0$ there exists a number $\alpha_{r}(x)<1$ such that

$$
\|x-y\| \leqq \alpha_{r}(x)\|(1+r)(x-y)-r(U(x)-U(y))\|, \quad y \in D .
$$

The following is another consequence of Theorem 1.

THEOREM 3. Let $X$ be a reflexive Banach space, $G$ a bounded open convex subset of $X$ with $0 \in G$, and suppose $U: X \rightarrow X$ is a lipschitzian strongly pseudo-contractive mapping relative to $\bar{G}$ satisfying

(i) $U(x) \neq \lambda x$ if $x \in \partial G, \lambda>1$.

Then $U$ has a fixed point in $\bar{G}$. 
Proof. In view of Theorem 1 we need only show that $(I-U)(\bar{G})$ is closed, and because $X$ is reflexive we need only show that $I-U$ is demiclosed. Thus if $u_{j} \rightarrow u_{0}$ weakly and if $(I-U)\left(u_{j}\right) \rightarrow w$ strongly then we must show $(I-U)\left(u_{0}\right)=w$. Defining $F: X \rightarrow X$ by $F(x)=U(x)+w$, then $F$ is lipschitzian and strongly pseudo-contractive on $X$ relative to $\bar{G}$, and furthermore $(I-F) u_{j} \rightarrow 0$ strongly. We show that this implies $u_{j} \rightarrow u_{0}$ strongly and this, with continuity of $U$, gives the desired result.

Choose $\lambda>0$ so small that $\lambda F$ is a contraction mapping with lipschitz constant $\lambda_{0}<1$, and let $r>0$ satisfy $\lambda=r /(r+1)$. Since $F$ is strongly pseudocontractive on $X$ relative to $\bar{G}$, for each $x \in X$ there exists $\alpha(x)$ such that

$$
\|x-y\| \leqq \alpha(x)\|(1+r)(x-y)-r(F(x)-F(y))\|, \quad y \in \bar{G} ;
$$

this is equivalent to

$$
(1-\lambda)\|x-y\| \leqq \alpha(x)\|x-y-\lambda(F(x)-F(y))\|,
$$

and since $(I-\lambda F)(X)=X$, we see that the mapping $F_{j}=I-\lambda F$ satisfies

(*) $\left\|(1-\lambda) F_{\lambda}^{-1}(x)-(1-\lambda) F_{i}^{-1}(y)\right\| \leqq \alpha(x)\|x-y\|, \quad x \in X, y \in \bar{G}$.

Now let $z_{j}=F_{\lambda} u_{j}$. Then

$$
\begin{aligned}
z_{j}-(1-\lambda) F_{\lambda}^{-1}\left(z_{j}\right) & =u_{j}-\lambda F\left(u_{j}\right)-(1-\lambda) u_{j} \\
& =-\lambda\left(F\left(u_{j}\right)-u_{j}\right) \rightarrow 0 \text { strongly. }
\end{aligned}
$$

The inequality $(*)$ permits us to complete the proof precisely as in Kirk [4] by showing that $\left\{z_{j}\right\}$ is necessarily a Cauchy sequence. (Replace $F$ with $(1-\lambda) F_{\lambda}^{-1}$ and $\left\{u_{j}\right\}$ with $\left\{z_{j}\right\}$ in [4]. Note the misprint in [4, p. 411]; one should have

$$
\left.C_{\xi}=\bigcup_{k=1}^{\infty}\left(\bigcap_{i=k}^{\infty} S\left(u_{j} ; \rho_{0}+\xi\right)\right) .\right)
$$

It follows that $\left\{u_{i}\right\}$ is a Cauchy sequence:

$$
\begin{aligned}
\left\|u_{i}-u_{j}\right\| & \leqq\left\|u_{i}-\lambda F\left(u_{i}\right)-\left(u_{j}-\lambda F\left(u_{j}\right)\right)\right\|+\left\|\lambda F\left(u_{i}\right)-\lambda F\left(u_{j}\right)\right\| \\
& \leqq\left\|z_{i}-z_{j}\right\|+\lambda_{0}\left\|u_{i}-u_{j}\right\|
\end{aligned}
$$

hence $\left(1-\lambda_{0}\right)\left\|u_{i}-u_{j}\right\| \leqq\left\|z_{i}-z_{j}\right\| \rightarrow 0$ as $i, j \rightarrow \infty$. Therefore $\left\{u_{j}\right\}$ converges strongly, and this completes the proof.

REMARK. We make one final observation. For $A \subset X$, let $\gamma(A)$ denote the measure of noncompactness of $A$ (cf. [5, p. 318]); $\gamma(A)=\inf \{d>0 \mid A$ can be covered by a finite number of sets of diameter less than or equal $d\}$. A continuous mapping $T: D \rightarrow X$ is said to be a $k$-set contraction, $k \geqq 0$, if 
for each bounded set $A$ in $D, \gamma(T(A)) \leqq k \gamma(A)$. Now suppose $U: D \rightarrow X$ satisfies

$$
\gamma(A) \leqq \gamma(((1+r) I-r U)(A)), \quad A \subset D, r>0 .
$$

For $S, T, H$ as defined in the proof of Theorem 1 , one can easily show that if $A \subset \bar{G}$ then $\gamma(S(A)) \leqq \gamma(T(A))$, and thus $\gamma(H(A)) \leqq \gamma(A)$. This proves that $H$ is a 1-set contraction, and application of Petryshyn's theorem [6] in its full generality to $H$ yields the fact that Theorem 1 holds for a more general class of mappings, namely the lipschitzian mappings which satisfy $(* *)$.

\section{REFERENCES}

1. F. E. Browder, Nonlinear mappings of nonexpansive and accretive type in Banach spaces, Bull. Amer. Math. Soc. 73 (1967), 875-882. MR 38 \#581.

2. - Semicontractive and semiaccretive nonlinear mappings in Banach spaces, Bull. Amer. Math. Soc. 74 (1968), 660-665. MR 37 \#5742.

3. W. A. Kirk, Remarks on pseudo-contractive mappings, Proc. Amer. Math. Soc. 25 (1970), 820-823. MR 41 \#9074.

4. - On nonlinear mappings of strongly semicontractive type, J. Math. Anal. Appl. 27 (1969), 409-412. MR 39 \#6128.

5. C. Kuratowski, Topologie. Vol. 1. Espaces métrisables, espaces complets, 2nd ed., Monografie Mat., vol. 20, PWN, Warsaw, 1948. MR 10, 389.

6. W. V. Petryshyn, Structure of the fixed points sets of $k$-set-contractions, Arch. Rational Mech. Anal. 40 (1970/71), 312-328. MR 42 \#8358.

Department of Mathematics, University of Iowa, Iowa City, Iowa 52240

Current address (Juan A. Gatica): Instituto de Matematico, Universidad de Concepción, Concepción, Chile 